\title{
Optimizing the FEI Volta Phase Plate for Efficient and Artefact-free Data Acquisition
}

\author{
Bart Buijsse $^{1}$, Radostin Danev ${ }^{2}$, Kasim Sader $^{1}$, Sonja Welsch $^{1}$ \\ 1. FEI, Achtseweg Noord 5, 5651 GG Eindhoven, The Netherlands \\ 2. Max Planck Institute of Biochemistry, Am Klopferspitz 18, Martinsried, Germany
}

FEI introduced the Volta Phase Plate (VPP) as a new product in 2014. It has been known for a long time that a phase plate should facilitate the observation of weak phase objects such as cryo-EM samples, but only with the introduction of the VPP have we started to exploit this advantage in practical applications. VPP-based tomography has revealed otherwise invisible particles, which could successfully be used in subtomogram averaging techniques [1]. VPP-based single particle work has enabled a near atomic reconstruction of a protein imaged close to focus [2]. The current insight is that VPP technology may provide answers in situations where conventional cryo-EM is reaching a limit, e.g. due to small particle size, or conformational heterogeneity of the particle.

Data acquisition with the VPP is still considered challenging by many users. There are some restrictions in the way the specimen illumination is set up, and any subsequent change of condenser optics may have consequences for the position of the focused electron beam on the phase plate. In addition, several other factors that have an impact on the focused beam position could be identified. Misalignment of beam and phase plate leads to artefacts in the contrast transfer of the specimen, or even a complete loss of VPPinduced contrast. FEI's challenge has been to facilitate use of the VPP in a manner that takes all these issues into account, while still keeping the data acquisition flow simple and efficient.

Apart from optical challenges, variation of VPP behavior between different production batches has also been observed. Initial phase plate characterization quantifies the contrast build-up in an image of amorphous carbon as a function of applied electron dose. This contrast can be compared with a defocused image without phase plate, and a characteristic dose can be defined for which the phase shift build-up is optimal. Similar information can be obtained from Thon ring fitting, although subtle charging effects can easily be overlooked in such a fit. Ultra-clean production methods and heating to sufficiently high temperatures have improved the phase shift development behavior of the phase plate.

In the alignment section of the FEI microscope UI we have introduced several new alignment steps to improve phase plate performance. In the phase plate alignments we have introduced methods based on the observation of a Ronchigram. This Ronchigram method helps to accurately set on-plane conditions and to determine the beam shift pivot points. Accurate setting of the beam shift pivot points is crucial for low-dose work, where un-tilted off-axis illumination conditions are often used. However, ronchigram observation for alignment purposes requires a fast camera. Where such a camera is not present, an alternative method based on beam scattering caused by the phase plate can be used.

In a 3-lens condenser system, flexibility for setting the on-plane condition is higher than in a 2-lens condenser system. For 3-lens systems, we have developed a method to keep the illuminated area constant, while refining the on-plane condition. Furthermore we introduced alignments for correction of small deviations of beam position on the phase plate as a result of focus changes.

In application software packages it is crucial to define a logical workflow for setting up different optical 
conditions, and defining the timing for phase plate activation. Typical problems that can occur are related to hysteresis in the optics, lens normalizations, sample charging, and focus changes. Solutions for avoiding these problems will be discussed.

For single particle work with the VPP, one may choose to work in-focus, or with the conventional defocus. In-focus work has the advantage that CTF correction can be avoided and that an uncompromised contrast transfer up to few Ångstrom resolution can be obtained. However, this requires precise focus determination, which makes improvements in the autofocus algorithm necessary.

Ideally, the contrast improvement delivered by the phase plate should be combined with the use of an electron counting detector. Electron counting provides the most sensitive detection method, but a low dose rate is required for imaging. However, at low dose rate, phase plate activation may take unacceptably long. In the presentation several solutions to this problem will be discussed.

\section{References:}

[1] S Asano et al, Science 347, p.439-442 (2015)

[2] M Khoshouei et al, Nature Communications 7, 10534 (2016)
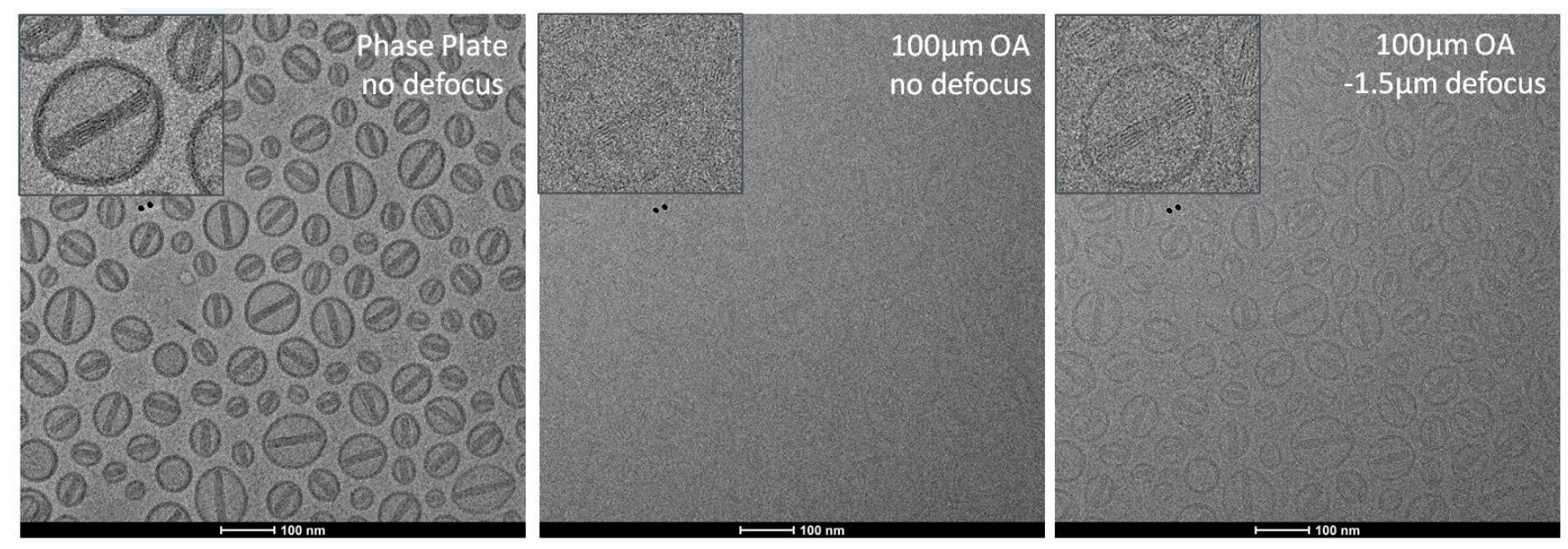

Figure 1. Doxil liposomes images acquired in-focus with Volta Phase Plate (left), in-focus with objective aperture (middle), and defocused with objective aperture (right). 